\title{
Preface
}

\section{Improving Sleep \\ Measurements for the Future}

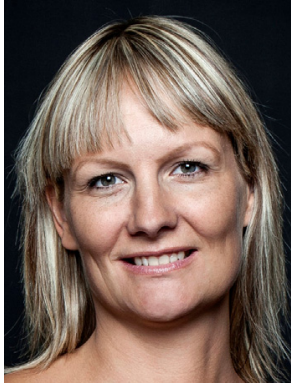

Erna Sif Arnardottir, PhD

Editor

The field of sleep is at a crossroads. Experts in sleep medicine and sleep research are calling out for new methods for both the diagnosis and treatment of different sleep disorders. We are realizing that the current state-of-the-art sleep measurement is not sufficient. We need to raise the bar and employ emerging technology to the fullest for the benefit of the numerous patients with sleep problems. This challenge is highlighted in the 10 excellent reviews in this issue on different aspects of sleep measurements. Importantly, both objective and subjective ways to measure sleep are discussed, as the role of the subjective sleep measurement is equally important as the objective one.

The need for sleep studies has increased exponentially in recent years as awareness for the importance of a good night's sleep has exploded among the general population and health care staff. Therefore, the current gold standard of inlaboratory polysomnography must be challenged and, in many cases, be moved to self-applied home sleep recordings to decrease the long waiting lists, workload, and costs of each sleep study. The heavy manual labor of scoring each sleep study also must be shortened for the same reasons. Finally, new parameters informing us better about the treatment need of individual patients than the current parameters are desperately needed. The role of interdisciplinary work, that is, working with experts in machine learning to move beyond the current state-of-the-art, is paramount to achieve these needed changes and to get more from sleep recordings than we currently do.
The role of daytime functioning, including objective cognitive performance and subjective measurements or patient-reported outcomes, is also of great importance. This field of sleep measurements needs substantial improvement to further understand how people are affected by sleep issues and to enhance patient care as reviewed in this issue. The role of wearable and digital health technology for our patient populations is another hot topic. The potential of this technology to complement traditional health care and research methods is huge. Sleep experts as a whole need to take advantage of this to the best of their abilities and perform the relevant research studies to validate their role. Finally, three different subgroups of patients are studied in more detail: women, children, and patients with neurologic disorders, to emphasize the need for different measurement tools and validations of such tools for different patient groups.

One size does indeed not fit all. Personalized sleep medicine, different treatment options as well as predictive, preventive, and participatory elements are all needed to push the boundaries of the sleep field. It is time for a revolution of sleep measurements!

Erna Sif Arnardottir, PhD Reykjavik University Sleep Institute School of Technology Reykjavik University Menntavegi 1 102 Reykjavik, Iceland

E-mail address: ernasifa@ru.is 